\title{
PLEURAL FLUID ADA IN THE DIAGNOSIS OF PLEURAL EFFUSION
}

\author{
Damanjit Duggal1, Anand Yannawar², Vinay Dharmadhikari ${ }^{3}$
}

${ }^{1}$ Associate Professor, Department of Respiratory Medicine, Dr. D. Y. Patil Medical College Hospital and Research Centre, Pimpri, Pune. ${ }^{2}$ Assistant Professor, Department of Respiratory Medicine, Dr. D. Y. Patil Medical College Hospital and Research Centre, Pimpri, Pune. ${ }^{3}$ Associate Professor, Department of Respiratory Medicine, Dr. D. Y. Patil Medical College Hospital and Research Centre, Pimpri, Pune.

\section{ABSTRACT}

\section{BACKGROUND}

Evaluation of pleural effusion is a common medical problem and despite the use of standard pleural fluid measurements and pleural biopsy the aetiology remains uncertain in a considerable number of cases. In our study, we assessed the importance of ADA activity in the diagnosis of pleural effusion.

\section{MATERIALS AND METHODS}

We studied 100 cases of pleural effusions of varying aetiology. Analysis of pleural fluid biochemistry cytology, ADA levels, ZN stain and results of pleural biopsy were analysed. This was a descriptive study. Statistical Analysis- Data were summarised using descriptive statistics such as percentages for categorical variables and mean with standard deviation for quantitative variables. For inferential statistics, two sample t-test was applied. A 'p' value of $<0.05$ was considered significant. Sensitivity, specificity, positive predictive value and negative predictive values were calculated for using ADA as a screening test of TB pleural effusion.

\section{RESULTS}

We found Tubercular and Parapneumonic effusions in a younger age group (20 - 50 yrs.), whereas malignant effusions and those due to cardiac failure were found in an older subset (50 - 80 yrs.). Pleural biopsy positivity rate was $60 \%-80 \%$ in tuberculosis and $50 \%-85 \%$ in malignant effusions. ZN smear for AFB gave poor yield in sputum and pleural fluid. In all our patients, pleural fluid ADA was above $41.5 \mathrm{U} / \mathrm{L}$ with a mean value of $73.92 \mathrm{U} / \mathrm{L}$.

\section{CONCLUSION}

Pleural fluid ADA in tubercular pleural effusion has a sensitivity, specificity, positive predictive value and negative predictive value at a cut-off of $41.5 \mathrm{U} / \mathrm{L}$ of $100 \%, 87 \%, 90 \%, 100 \%$ respectively.

\section{KEYWORDS}

Pleural Effusion, ADA, Tuberculosis.

HOW TO CITE THIS ARTICLE: Duggal D, Yannawar A, Dharmadhikari V. Pleural fluid ADA in the diagnosis of pleural effusion. J. Evolution Med. Dent. Sci. 2017;6(60):4421-4429, DOI: 10.14260/Jemds/2017/956
BACKGROUND
Evaluation of pleural effusion is a common medical problem and despite the use of standard pleural fluid measurements,
1. Pleural fluid to serum protein ratio $>0.5$.
2. Pleural fluid LDH level $>200$ IU.
3. Pleural fluid $\mathrm{LDH}$ to serum $\mathrm{LDH}$ ratio $>0.6$. the aetiology remains uncertain in some cases. Even after diagnostic investigation of pleural fluid and closed needle biopsy of pleura, the cause is sometimes perplexing. Tuberculosis is still one of the most important cause of exudative pleural effusion. The diagnosis of tuberculosis should be considered in any patient with an exudative pleural effusion. Many of the undiagnosed cases of pleural effusion are of tubercular aetiology.

A single chemical test or a set of chemical tests rarely give $100 \%$ effective results to separate transudative from exudative fluid, but increasing the numbers of test results in a more reliable separation. The simultaneous use of pleural fluid protein and LDH levels is more effective than the use of either one itself. The presence of any one of the following three characteristics indicate that the fluid is an exudate.

Financial or Other, Competing Interest: None.

Submission 08-05-2017, Peer Review 14-07-2017,

Acceptance 20-07-2017, Published 27-07-2017.

Corresponding Author:

Dr. Damanjit Duggal,

Department of Respiratory Medicine,

Dr. D. Y. Patil Medical College Hospital and Research Centre,

Pimpri, Pune.

E-mail: drdamanjitduggal@yahoo.co.in

DOI: $10.14260 /$ jemds $/ 2017 / 956$

Routine Investigations as follows on Pleural Fluid does not give any Aetiological Diagnoses

1. Pleural fluid colour, presence of cobweb/coagulum.

2. Pleural fluid protein content.

3. Specific gravity of pleural fluid and $\mathrm{pH}$.

4. Pleural fluid glucose level.

5. Pleural fluid cytology.

Additional investigation like pleural fluid amylase, cholesterol, LDH, tumour markers and Adenosine Deaminase (ADA) may help in reaching specific diagnosis. Many workers found very low yield of pleural fluid on direct smear examination for AFB (Z-N Stain).(1,2)

\section{Pleural Fluid Culture for Mycobacterium Tuberculosis}

In most series of tuberculous pleurisy, positive mycobacterial culture of pleural fluid was obtained in less than $25 \%$ cases. The percentage of positive culture has been increased by centrifugation of large amount of pleural fluid, multiple cultures and guinea pig inoculation. The liquid media are superior to Lowenstein-Jensen media.(2) Magath et al (1932); Oshima et al (1933); Eberle et al (1949); Willis and Cummings (1952); Ives and Macormick et al (1956); Falk et al (1950) discussing idiopathic pleural effusion thought that 
routine culture and guinea pig inoculation should be expected to be positive in $20 \%-30 \%$ of large number of cases.(1)

\section{Pleural Biopsy}

Pleural biopsy can be studied for histopathology; AFB culture and fungal culture. It often gives a rapid and accurate diagnosis when other methods fail. But it should be emphasised that the tubercles and metastasis may be distributed on pleura in a patchy fashion and the sample obtained may not be from the pathological tissue. As the patient of pleural effusion lies down, most of the times the fluid collects in posterior part of pleural cavity and hence this region is more likely to show tubercular changes than other regions. Multiple specimens from the same site gives more positive results.(2) The pleural biopsy is associated with morbidity and is not diagnostic in each and every patient of pleural effusion. It is contraindicated in bleeding diathesis and empyema. Due to those apparent disadvantages a search for more simple and useful method became essential.

\section{MATERIALS AND METHODS}

\section{Study Sample and Setting}

This descriptive study consisted of 100 consecutive patients. For convenience 100 patients were studied who met inclusion criteria and were admitted in a tertiary care centre over a period of 3 years.

There were 80 male and 20 female patients. The youngest patient was 17 years old male and the oldest was 87 years old male patient.

\section{Inclusion Criteria were as Follows}

1. Tubercular pleural effusion
a. Sputum positive.
b. Pleural biopsy positive for tuberculosis.
c. TB-PCR for AFB positive.
d. AFB present in pleural fluid.

2. Malignant pleural effusion

a. Malignancy proven on pleural biopsy.

b. Pleural fluid PAP smear positive.

3. Parapneumonic pleural effusion.
a. Sputum positive for Gram statin.
b. Pleural fluid positive for gram stain.
c. Frank pus in pleural fluid.
d. Pleural biopsy (VATS) s/o empyema.

4. Liver cirrhosis

- Liver cirrhosis proven on USG.

- Deranged Liver function test.

5. Renal failure

- Biopsy proven ESRD/Glomerulonephritis/Nephrotic syndrome.

- Patient on dialysis.

6. Pleural effusion secondary to congestive cardiac failure

- Clinical diagnosis.

- 2-D Echo diagnosis.

- BNP levels.
7. Post $\mathrm{CABG}$ pleural effusion

- Diagnosis of exclusion.

- Light's criteria for diagnosis.

8. Pleural effusion secondary to pancreatitis

- Pleural fluid amylase.

- Exclusion of other diagnosis.

9. Interstitial lung diseases

- HRCT criteria s/o ILD.

- Diagnosis of exclusion.

\section{Following Methods were Adopted}

In all cases of pleural effusion, diagnostic and therapeutic (as per need) thoracentesis was achieved. Pleural fluid was analysed for biochemistry, microbiology and cytology. Special investigations like TB-PCR was also done in suspected cases. In some cases, with multiple loculations, thoracoscopy was done for adhesiolysis and pleural biopsy. In suspected malignant cases, diagnostic thoracoscopy was done with pleurodesis. Diagnostic aspiration was performed in confirmed cases of congestive cardiac failure, liver cirrhosis and renal failure.

The Following Investigations were done in all Pleural Fluids Initially

1. Biochemistry: - Sugar, Protein and ADA.

2. Microbiology: - Gm, Z-N stain.

3. Cytology: - For malignant cells.

On Analysis of these data Further Required Investigations and Interventions were done

1. TB- PCR.

2. Pleural Biopsy.

3. Thoracoscopy (VATS) adhesiolysis, pleurodesis and pleural biopsy.

Histopathological Appearances of Pleural Biopsy was classified as

1. Tubercular inflammation.

2. Nonspecific chronic inflammation.

3. Malignancy.

The Accepted Criteria for Positive Pleural Biopsy were

1. Tubercular inflammation- Typical changes of tuberculosis were collection of epithelioid cells with or without caseation and Langhans giant cells.

2. Nonspecific chronic inflammation- Necrotic material with polymorphs and lymphocytes with fibroblastic reaction.

3. Malignancy- Cells with hyperchromatic nuclei infiltrating in between muscle bundles may be anaplastic or differentiated.

The Separation of the Pleural Fluid as Transudate or Exudates was done on the Basis of

1. Pleural fluid protein content.

2. Pleural fluid to serum protein ratio.

\section{ADA Estimation in Pleural Fluid}

ADA activity was established in all the samples of pleural fluid collected from all 100 patients by the sensitive colorimetric methods described by Giusti (1974).(3) 


\section{Statistical Analysis}

This was a descriptive study. Data were summarised using descriptive statistics, such as percentages for categorical variables and mean with standard deviation for quantitative variables. For inferential statistics, two sample t-test was applied. A ' $p$ ' value of $<0.05$ was considered significant. Sensitivity, specificity, positive predictive value and negative predictive values were calculated for using ADA as a screening test of TB pleural effusion. Pleural fluid ADA value of $41.5 \mathrm{U} / \mathrm{L}$ was used as a cut-off for tubercular pleural effusion.

\section{RESULTS}

\section{Age vs Disease Distribution}

Tubercular effusion and parapneumonic effusion predominates among young and middle age group (20 - 50 years) than those with malignant effusion and cardiac failure (50 - 80 years).

\section{Sex Distribution of Patient}

There were 20 females and 80 males.

\section{Diagnostic Yield of Sputum in TB Pleural Effusion}

In present study yield of sputum in diagnosis of tuberculosis was very low, only 15 cases out of 54 were positive for AFB (27\%), 34 samples were negative for AFB (62\%), remaining 5 out of 54 had unproductive cough.

\section{Diagnostic Yield of Pleural Biopsy in TB and Pleural Malignancy}

Pleural biopsy in present study had better yield, $70 \%$ in tuberculosis effusion and 95\% in malignant effusion.

\section{Diagnostic Yield of Pleural Fluid Z-N Staining in TB}

$90 \%$ of patients were found out to be negative and only $10 \%$ of patients were found out to be positive.

\section{Diagnostic Yield of Pleural Fluid Cytology in Malignant Effusion}

Diagnostic yield in malignant pleural effusion was found out to be positive in $68.42 \%$ cases and negative in $31.58 \%$ cases in a sample of 19 patients.

\section{Distribution of Pleural Effusion in TB}

Pleural fluid distribution of tuberculous effusion, malignant and others was also studied in present study. The tuberculous pleural effusion was on right side in $50 \%$ of patients, on left side in $44 \%$ of patients and on both sides in $6 \%$ of patients. All congestive cardiac failure patients had effusion on both sides, chronic renal failure patients on dialysis also showed same results.

\section{Distribution of Pleural Fluid in Case of Malignant Effusion} Malignant pleural effusion also had same distribution with right sided predominance $(63.15 \%)$, left sided in $26.31 \%$ and bilateral in remaining (10.53\%).

\section{Distribution of Pleural Fluid in Remaining Cases}

Pleural effusion in a case of cardiac failure and renal failure was found to be bilateral. While in Liver Cirrhosis it was unpredictable since in two cases it was right pleural effusion, while in three cases it was left pleural effusion. Other patients did not have any particular side dominance for effusion and sample size was also too small to make comments.

\begin{tabular}{|c|c|c|c|c|}
\hline Group & $\begin{array}{c}\text { No. of } \\
\text { Cases (\%) }\end{array}$ & $\begin{array}{c}\text { Mean ADA } \\
\text { Value }\end{array}$ & $\begin{array}{c}\text { Standard } \\
\text { Deviation }\end{array}$ & Range \\
\hline $\begin{array}{c}\text { Tuberculous } \\
\text { Pleural } \\
\text { Effusion }\end{array}$ & 54 (54\%) & 73.92 & 33.95 & $\begin{array}{c}41.5- \\
139.7\end{array}$ \\
\hline $\begin{array}{c}\text { Malignant } \\
\text { Pleural } \\
\text { Effusion }\end{array}$ & $19(19 \%)$ & 18.83 & 10.53 & $5.1-53.6$ \\
\hline $\begin{array}{c}\text { Pleural } \\
\text { Effusion } \\
\text { Secondary to } \\
\text { Congestive } \\
\text { Cardiac } \\
\text { Failure }\end{array}$ & $7(7 \%)$ & 11.45 & 5.63 & $1.17-18$ \\
\hline $\begin{array}{c}\text { Pleural } \\
\text { Effusion due } \\
\text { to Chronic } \\
\text { Renal Failure }\end{array}$ & $5(5 \%)$ & 16.38 & 5.25 & $11.0-23.0$ \\
\hline $\begin{array}{c}\text { Pleural } \\
\text { Effusion due to } \\
\text { Liver Cirrhosis }\end{array}$ & $5(5 \%)$ & 17.12 & 3.88 & $15.0-24.0$ \\
\hline $\begin{array}{c}\text { Para- } \\
\text { pneumonic } \\
\text { Effusion }\end{array}$ & $4(4 \%)$ & 32.12 & 14.33 & $11.6-45$ \\
\hline $\begin{array}{c}\text { Post CABG } \\
\text { Pleural } \\
\text { Effusion }\end{array}$ & $3(3 \%)$ & 20.743 & 19.3 & $0.54-39$ \\
\hline $\begin{array}{c}\text { Pancreatitis, } \\
\text { Pleural } \\
\text { Effusion }\end{array}$ & $2(2 \%)$ & 23.5 & 0.7 & $23-24$ \\
\hline $\begin{array}{c}\text { Interstitial } \\
\text { Lung Disease }\end{array}$ & $1(1 \%)$ & 4.59 & & \\
\hline \begin{tabular}{c} 
Table 1. Analysis of ADA in TB and in Other Diseases \\
\hline
\end{tabular} & & & & \\
\hline
\end{tabular}

Table 1 shows range of ADA values for different diseases and their standard deviation from mean. From above table, we can conclude that range of ADA for Tuberculous Pleural Effusion is much higher than in other diseases. Standard deviation is also high. This may be due to changing observation environment or due to less accuracy of machine.

\begin{tabular}{|c|c|c|}
\hline & $\begin{array}{c}\text { ADA values in TB } \\
(\mathbf{n = 5 4 )}\end{array}$ & $\begin{array}{c}\text { ADA values in } \\
\text { Malignancy (n= 19) }\end{array}$ \\
\hline Mean & 73.92 & 18.27 \\
\hline $\begin{array}{c}\text { Standard } \\
\text { Deviation }\end{array}$ & 33.95 & 10.53 \\
\hline T Statistic & 11.25 & \\
\hline $\begin{array}{c}\text { P (T<= t) two- } \\
\text { tail }\end{array}$ & $<0.001$ & \\
\hline
\end{tabular}

Table 2. Comparison of ADA- Tuberculous Pleural Effusion and Malignant Pleural Effusion

Table 2 shows that there is high and significant difference in the ADA values between TB effusion and malignancy with absolute difference of over 50 units. 


\begin{tabular}{|c|c|c|}
\hline & $\begin{array}{c}\text { ADA values in TB } \\
(\mathbf{n = 5 4 )}\end{array}$ & $\begin{array}{c}\text { ADA values in Cardiac } \\
\text { Failure (n= 7) }\end{array}$ \\
\hline Mean & 73.92 & 11.45 \\
\hline $\begin{array}{c}\text { Standard } \\
\text { Deviation }\end{array}$ & 33.95 & 5.63 \\
\hline T Stat & 12.98 & \\
\hline $\begin{array}{c}\text { P }(\mathrm{T}<=\mathrm{t}) \\
\text { two-tail }\end{array}$ & $3.07688 \mathrm{E}-18$ & \\
\hline $\begin{array}{c}\text { T Critical } \\
\text { two-tail }\end{array}$ & 2.00 & \\
\hline
\end{tabular}

Table 3. Tuberculous Pleural Effusion and Pleural Effusion Secondary to Congestive Cardiac Failure

Table 3 shows that ADA values in TB is about 60 units higher compared to effusion in congestive heart failure. This difference was statistically highly significant.

Table 1 shows that ADA levels in TB pleural effusion was much higher compared to effusion in renal failure. Similarly, Table 1 shows that ADA values in TB effusion was much higher than effusion in cirrhosis. Also in patients with parapneumonic effusion and post-CABG cases with pleural effusion had much lower ADA values. Since sample size was too small for these cases, no test of statistical significance was applied.

\begin{tabular}{|c|c|c|c|}
\hline Screening Test & Tuberculosis & \begin{tabular}{|c|} 
Non- \\
Tuberculosis \\
\end{tabular} & Total \\
\hline Positive & 54 & 6 & 60 \\
\hline Negative & 0 & 40 & 40 \\
\hline Total & 54 & 46 & 100 \\
\hline
\end{tabular}

From above table, we can calculate value of sensitivity and specificity. Therefore, Sensitivity of Tuberculous Pleural Effusion is $100 \%$ and Specificity comes out to be $87 \%$. Positive predictive value $90 \%$ and Negative predictive value $100 \%$.

\section{DISCUSSION}

A systematic approach to the classification of pleural effusion is needed because of extensive differential diagnosis. Diagnostic evaluation is based on the analysis of clinical variables (gender, age and symptoms), imaging (chest x-ray, USG of chest) and laboratory analysis of blood and pleural fluid. Tubercular effusion is the common cause of exudative pleural effusion in many areas of the world, which is consistent with my study which shows that 54 patients were having tubercular effusion out of 100 . Tubercular effusion and parapneumonic effusion predominates among young and middle age people (20 - 50 years) than those with malignant effusion and cardiac failure (50 - 80 years), this fact is also proven by present study.

A predominance of neutrophils in the pleural fluid (more than 50 percent of the cells) indicates that an acute process is affecting the pleura. Light RW et al in a series found 21 of 26 parapneumonic effusions (81 percent), 4 of 5 effusions secondary to pulmonary embolus (80 percent) and 4 of 5 effusions secondary to pancreatitis ( 80 percent) contained more than 50 percent neutrophils, but only 7 of 43 malignant effusions (16 percent) and none of 14 tuberculous effusions contained more than 50 percent neutrophils. Present study also shows that in parapneumonic effusion, total neutrophil count is above $50 \%$ in all cases which shows that parapneumonic effusion is an acute process affecting pleura.

A preponderance of lymphocytes indicate that the patient most likely has cancer or tuberculous pleuritis, although such a preponderance is also seen in pleural effusions after coronary artery bypass surgery.(4) The combined data from two series, Keshmiri M et al(5) and Fine et al(6) show that 90 of 96 exudative pleural effusions consisting of more than 50 percent lymphocytes (94 percent) were due to cancer or tuberculosis. Yam LT et al(7) had case series of 116 patients, 90 of 116 tuberculous pleural effusions (78 percent) contained more than 50 percent lymphocytes. Present study had similar results with lymphocyte count was more than $50 \%$ in $90 \%$ cases ( 49 out of 54 ), neutrophils predominates in $10 \%$ of cases ( 5 out of 54 ), all these patients had a history of repeated pleural tapping. Neutrophils predominance may be due to iatrogenic or bacterial infection. Lymphocytes predominance was seen in $100 \%$ malignant effusion. Present study proves that there is no correlation between lymphocytes and underlying disease process. All patients have predominance of lymphocytes except parapneumonic effusion.

In present study yield of sputum in diagnosis of tuberculosis is very low, only 15 cases out of 54 were positive for AFB (27\%), 34 samples were negative for AFB (62\%), remaining 5 out of 54 have unproductive cough.

Diagnostic yield of pleural fluid Z-N staining is mentioned in observation charts. We can conclude that in TB there is more probability of diagnostic yield to be negative as from the sample space of $54,90 \%$ of patients were found out to be negative, while approximately $10 \%$ of patients were found out to be positive.

Pleural biopsy also fails to give diagnosis in all cases of tuberculosis and malignancy. In most of the studies, positivity rate for pleural biopsy varies from $60 \%-80 \%$ in tubercular pleural effusion and 50\% - 85\% in malignant pleural effusion. This diagnostic yield varies and depends upon number of attempts, clinical skills, experience and instruments (video thoracoscopy vs pleural biopsy needle) used. The disadvantage of pleural biopsy is it cannot be performed in every patient and requires specialised needles and expertise. Lights et al(8) had case series of 248 patients with tuberculous pleuritis who underwent needle biopsy of pleura. The biopsy showed granulomas in 198 patients (80\%), the acid fast stain of the biopsy tissue was positive in 140 (56\%). Pleural biopsy in present study had better yield, $70 \%$ in tuberculous effusion and $95 \%$ in malignant effusion.

The percentage of cases in which cytology of pleural fluid establishes diagnosis of malignant pleural effusion ranges from $40 \%$ to $87 \%$. In present study diagnostic yield of pleural fluid cytology was $68.42 \%$, comparable results as previous studies. $(9,10)$

Present study concludes that tubercular effusion is the commonest cause of unilateral pleural effusion followed by Parapneumonic effusion and congestive heart failure is the commonest cause of bilateral pleural effusion.

Pleural fluid distribution of tuberculous effusion, malignant and others was also studied in present study. The tuberculous pleural effusion was on right side in $50 \%$ of patients, on left side in $44 \%$ of patients and on both sides in $6 \%$ of patients. Malignant pleural effusion also had same distribution with right sided predominance (63.15\%), left 
sided in $26.31 \%$ and bilateral in remaining (10.53\%). All congestive cardiac failure patients had effusion on both sides, chronic renal failure patients on dialysis also showed same results. Other patients did not have any particular side dominance for effusion and sample size was also too small to make comments.

Several reports have suggested that an elevated pleural fluid ADA level predicts tuberculous pleuritis with a sensitivity ranging from $66 \%$ to $100 \%$ and a specificity of $59 \%$ to $100 \%$. Different authors have used various cut-off levels for the pleural fluid ADA between 30 and $70 \mathrm{U} / \mathrm{L}$ for the diagnosis of pleural TB. The higher the pleural fluid ADA level, the more likely the patient is to have tuberculous pleuritis. In a meta-analysis of 40 articles, the sensitivity and specificity of pleural fluid ADA levels in the diagnosis of pleural TB were both $92 \%$.(11) $^{(11}$

In general, the two main diseases other than tuberculous pleuritis that are associated with a high pleural fluid ADA are empyema and rheumatoid pleuritis. Present study had four cases of empyema, out of four cases three patients had pleural fluid ADA above 30. We did not find any case of rheumatoid pleuritis in our study. However, it should be easy to differentiate these two diseases from tuberculous pleuritis by the clinical picture and the fact that these latter two diseases do not have pleural fluid lymphocytosis. Indeed, if the diagnostic criteria for tuberculous pleuritis includes a pleural fluid lymphocyte-to-neutrophil ratio of 0.75 or more, the specificity of the test is increased.

Almost all patients with tuberculous pleuritis have an ADA level above $40 \mathrm{U} / \mathrm{L}$.

Immunosuppressed patients with tuberculous pleuritis have elevated pleural fluid ADA levels. The levels of ADA in patients with and without AIDS are comparable.(12) Renal transplant patients who develop a pleural effusion have elevated pleural fluid ADA levels.(13) Present study also had five cases of pleural effusion secondary to renal failure, but none of them had ADA value above $25 \mathrm{IU} / \mathrm{L}$.

The pleural fluid ADA level can be used to exclude the diagnosis of tuberculous pleural effusions in patients with undiagnosed pleural effusions. Piras et al (1978)(14) reported ADA concentration in 96 patients of effusion, of them 54 had pleural effusion and 42 had peritoneal effusion. The pleural effusions were due to tuberculous pleurisy (21 cases); parapneumonic effusion (15 cases); primary and metastatic pleura-pulmonary malignancy (18 cases). The peritoneal effusions were due to liver cirrhosis and ascites (25 cases) and abdominal primary and metastatic malignancy (17 cases). The mean ADA concentration in TB pleural effusion was $(83.04 \pm 25.51 \mathrm{IU} / \mathrm{L})$. This was higher than in parapneumonic pleurisy $(17.26 \pm 7.51 \mathrm{IU} / \mathrm{L})$ and pleurapulmonary malignancy $(15.54 \pm 6.74 \mathrm{IU} / \mathrm{L})$. The result of present study were also the same.

Sinha and Sinha et al (1985)(15) studied 36 patients. They did the comparative study to diagnose the pleural effusion by sensitivity of mycobacterial culture, histopathological and ADA activity. Bacteriological and histopathological evidence of tuberculosis was obtained in 22 patients (61\%); 5 patients (14\%) had parapneumonic effusion and $4(11 \%)$ had pleurapulmonary malignancy. The remaining 5 (14\%)- no definite diagnosis could be decided. The specimen for culture was ground in Teflon tissue grinder and inoculated in blood agar, in thioglycollate broth and on two slopes of Lowenstein-
Jensen culture medium. The slopes were incubated horizontally for one week and vertically for eight weeks. All positive cultures were examined microscopically and tested for niacin production to confirm the presence of acid fast bacilli. Among 22 patients of tuberculosis pleural tissue mycobacterial culture was positive in $77 \%$; histopathology showed granuloma in 55\%. Pleural fluid smear was positive in 1 and sputum smear for AFB was positive in 2 patients. They found mean ADA activity in tubercular pleural effusion as $76.8 \pm 23.8 \mathrm{IU} / \mathrm{L}$ as compared to 5 patients with parapneumonic effusion $15.2 \pm 5.1 \mathrm{IU} / \mathrm{L}$ and patients with pleura-pulmonary malignancy $14.5 \pm 8$.9I U/L.

Most current laboratory tests like pleural tissue culture and histopathology are slow, invasive and comparatively less sensitive and their results depends on the availability of expert's opinion. Significantly, raised ADA concentration in the pleural fluid of patients with tuberculous pleurisy suggests that ADA activity in laboratory may be a rapid, simple and sensitive laboratory tests for the diagnosis or confirmation of tuberculous pleural effusion.

Rajendra Prasad et al (1989)(16) also found high ADA activity in pleural fluid of patients with tubercular pleural effusion as compared with malignant effusion, pneumonic effusion and transudative pleural effusion.

Ferrer et al(17) followed up 40 patients with undiagnosed pleural effusions and a pleural fluid ADA level below $43 \mathrm{U} / \mathrm{L}$ for a mean of 5 years and reported that none of the patients developed TB. Lymphocytic pleural effusions, not due to tuberculous pleuritis usually have pleural fluid ADA levels below $40 \mathrm{U} / \mathrm{L}$.

In present study, all tuberculous pleural effusions had ADA value of above $41.5 \mathrm{U} / \mathrm{L}$.

Comparative study for pleural biopsy and ADA by B. R. Maldhure et al (1993)(18) with 125 cases revealed that out of 105 pleural biopsy $36(45.56 \%)$ cases are definitely tubercular and 17 (21.51\%) had suspected findings. ADA levels in pleural fluid were significantly raised in clinically diagnosed as well as in biopsy confirmed cases of tubercular pleural effusion. Also serum ADA levels were significantly raised. No significant difference was found between ratio of pleural fluid and serum ADA. Best cut-off point of pleural fluid ADA level for the diagnosis of tubercular pleural effusion was found to be $60 \mathrm{U} / \mathrm{L}$ (15) with $60.32 \%$ specificity, $80.55 \%$ sensitivity, $52.73 \%$ positive predictive value and $80 \%$ negative predictive value. The finding was confirmed on ROC curve.

Valdes (1996) ${ }^{(19)}$ also considered whether the results throw light on the origin of high pleural fluid ADA in tuberculous pleurisy and estimated the diagnostic efficiency of 2-deoxyadenosine deaminase, ADA-2 and total ADA activities. Valdes divided 350 pleural effusions as transudates (60 males/18 females) or as tuberculous (49 males/27 females), neoplastic (50 males/39 females), parapneumonic (36 males/19 females), empyematous (11 males/3 females), or miscellaneous ( 25 males/13 females) exudates. The total ADA in tuberculous effusion was $127.5 \pm 2.9 \mathrm{IU} / \mathrm{L}$ with cut-off value 47 , he had sensitivity and specificity of $100 \%$ and $91 \%$ respectively. As he considered ADA-2, which is more specific for tuberculous pleural effusion that is why he had better sensitivity and specificity. Valdes also concluded that ADA-2 originates from monocytes. ADA-2 was not considered in 
present study, as this investigation was more expensive and not easily available.

F. J. Al-Shammary (1997)(20) proved that pleural fluid ADA levels were very high in tuberculous pleural effusion as compared to non-tuberculous lymphocyte predominant pleural effusion. He also studied serum ADA value in tuberculosis, which was also high. Serum ADA value was not considered in present study.

Perez-Rodriquez E et al (2000)(21) determined ADA-1 and ADA-2 value in tuberculous pleural effusion. He also had same conclusions as Valdes results. ADA-2 is more specific for tuberculous effusion and originates from monocytes. He also calculated lymphocytes to neutrophils ratio to differentiate parapneumonic effusion from TB. ADA-2 and ratio was not studied in present study.

In our lab and clinical settings, tuberculosis effusion is strongly suspected if pleural fluid ADA level is above $40 \mathrm{U} / \mathrm{L}$. Mean value of ADA in tuberculous effusion is $73.92 \mathrm{U} / \mathrm{L}$ in present study. However, ADA may be falsely positive in few conditions. $(5,6,7)$ In present study also very few cases of malignant pleural effusion had borderline high ADA level.

In present study, the sensitivity and specificity of ADA is $100 \%$ and $87 \%$ at cut-off value of $41.5 \mathrm{IU} / \mathrm{L}$. Sharma et al $(2001)^{(22)}$ had a sensitivity and specificity of $83.3 \%$ and $66.6 \%$ respectively at a cut-off value of $35 \mathrm{IU} / \mathrm{L}$. There was increase in specificity with compromise in sensitivity at a cutoff value of $100 \mathrm{IU} / \mathrm{L}$. Present study had a higher sensitivity and specificity at a lower cut-off ADA value as compared to Sharma et al study. From this study, it is concluded that using $100 \mathrm{IU} / \mathrm{L}$ as the cut-off, it is possible to avoid pleural biopsy to ascertain the diagnosis of TB in as much as $40 \%$ of the patients. Present study also had higher ADA values in TB pleural effusion as compared to non-TB pleural effusion.

D. Jimenez Castro et al (2002)(23) selected 410 nontuberculous lymphocytic pleural fluid samples. These included malignant effusions $(n=221)$, idiopathic effusions $(n=76)$, parapneumonic effusions $(n=35)$, post-coronary artery bypass graft surgery effusions $(n=6)$, miscellaneous exudative effusions $(n=21)$ and transudative effusions $(n=$ 51). The ADA level reached the diagnostic cut-off for tuberculosis ( $40 \mathrm{U} / \mathrm{L}$ ) in seven of the 410 cases $(1.71 \%)$. The negative predictive value of ADA for the diagnosis of pleural tuberculosis was 99\% (403 of 407 cases) in the group of lymphocytic pleural effusions. This prospective study provides additional evidence that adenosine deaminase levels in non-tuberculous lymphocytic pleural effusions seldom exceed the cut-off set for tuberculous effusions. The pleural fluid adenosine deaminase levels were significantly higher in different types of exudative effusions than in transudates. An adenosine deaminase level $<40$ IU/L virtually excluded a diagnosis of tuberculosis in lymphocytic pleural effusions.

Jae Ho Chung et al (2004)(13) analysed the ADA activity in the pleural fluid of renal transplant recipients. He studied 23 renal transplant patients with tuberculous pleural effusion (21 men and 2 women, mean age 33 years). They were treated at the Yonsei University Hospital between January 1985 and December 2001. Patients with granuloma in the pleural biopsy specimen or positive for mycobacterial tuberculosis in the pleural effusion of 23 renal transplant patients with tuberculous pleural effusion was compared with 23 immunocompetent patients with TPE. The mean activity was $69.5 \pm 4.645$ in renal transplant patients and 65.0 $\pm 4.9 \mathrm{U} / \mathrm{L}$ in immunocompetent patients. Applying the $40 \mathrm{U} / \mathrm{L}$ 45 cut-off point, the positivity of ADA was $91.3 \%, 45$ in renal transplant patients and $86.9 \%$ in immunocompetent patients. He thus concluded that the measurement of ADA in the pleural fluid is the useful means in diagnosis of TPE in renal transplant patients.

Sh. Taghipour Zahir et al (2007)(24) performed analytical cross-sectional study of patients who were hospitalised in the infectious and internal wards on suspicion of tuberculosis or malignancy. First, pleural fluid was aspirated to measure the ADA level (cut-off point $35 \mathrm{U} / \mathrm{L}$ ) 53. Then biopsy was done and the results were compared with each other. Sh. Taghipour analysed data using SPSS 10 software and Chisquare test. Compared to biopsy, the sensitivity and specificity of ADA for the diagnosis of tuberculosis were $68.4 \%$ and $92.6 \%$ and for malignancies were $91.3 \%$ and $37.8 \%$ respectively. In comparison to biopsy, the positive predictive value of $\mathrm{ADA}$ to diagnose tuberculosis and malignancies was $81.2 \%$ and $47.8 \%$ respectively, and the negative predictive value of ADA for the diagnosis of TB and malignancies was $86.36 \%$ and $87.5 \%$ respectively. Based on the obtained data, he concluded that raised levels of ADA in pleural effusion (over $35 \mathrm{U} / \mathrm{L}$ ) suggest tuberculosis with more than $90 \%$ probability. Although low levels of ADA (below $35 \mathrm{U} / \mathrm{L}$ ) have great sensitivity for the diagnosis of malignancies, as the specificity of ADA level is $37.8 \%$ it does not necessarily propound malignancy.

Kamaldeen Baba et al (2008)(25) conducted a retrospective case control study. He revived medical files of patients with tuberculous pleuritis and non-tuberculous pleuritis. Clinical characteristics, CD 4 cell counts in blood and biochemical markers in pleural fluid including ADA were recorded. Kamaldeen evaluated 197 tuberculous pleuritis and 40 non-tuberculous pleuritis patients. Using the cut-off value of $30 \mathrm{U} / \mathrm{L}$ the overall sensitivity, specificity, positive likelihood ratio and negative likelihood ratio of ADA was 94\%, 95\%, 19 and 0.06 respectively. The mean CD4 cell counts among TB pleuritis patients was 29 and 153 cells/microL in patients with non-tuberculous effusion, 50 cells/microL and 50 cells/microL (p- 0.05) respectively. The corresponding mean ADA values for these patients were 76 $\mathrm{U} / \mathrm{L}$ and $72 \mathrm{U} / \mathrm{L}$ respectively $(\mathrm{p}-0.5)$. There was no correlation between ADA values and CD4 cell counts ( $r=$ 20.120, $\mathrm{p}=0.369$ ). He concluded that ADA analysis is a sensitive marker of tuberculous pleuritis even in HIV patients with very low CD4 counts in a high TB endemic region. The ADA assay is inexpensive, rapid and simple to perform and is of great value for the immediate diagnosis of tuberculous pleuritis while waiting for culture result and this has a positive impact on patient outcome.

Ashish Jadhav et al (2007)(26) performed a study on sixty indoor patients, admitted to hospital having pleural effusions and of varying aetiologies. According to the final diagnosis, he divided these 60 patients into two groups: exudates (50) and transudates.(9) The mean pleural ADA, serum ADA and pleural fluid/serum ADA ratio were significantly $(\mathrm{p}<0.0001)$ higher in exudates as compared to transudates. Using a cut-off point of $22 \mathrm{IU} / \mathrm{L}$, the sensitivity and specificity of pleural ADA in the diagnosis of exudates was computed to be $90 \%$ and $90 \%$ respectively. At a cut-off point 1.28, pleural fluid/serum ADA ratio was found to have sensitivity of $84 \%$ and specificity of 
$90 \%$, respectively. From this study he concluded that ADA is a useful biochemical marker to suggest exudative effusions.

In Dhital KR(27) study pleural fluid mean ADA value in tuberculous, malignant, congestive cardiac failure and renal effusions were 181.37, 52, 16 and $12 \mathrm{IU} / \mathrm{L}$, in present study the ADA levels were 73.92, 18.83, 11.45 and $16.38 \mathrm{IU} / \mathrm{L}$ respectively. The mean ADA levels in present study were much lower as compared to Dhital et al, but sensitivity and specificity were comparable to remaining reference studies.

Perlat Kapisyzi et al(28) studied the use of cut-off values of fluid ADA level in differential diagnosis of tuberculous pleurisy in country with low incidence of tuberculosis; 121 consecutive patients with pleural effusion were prospectively studied. The study included 82 males, 39 females with a mean age of $59.3 \pm 17$ years. Exudative effusions were classifiedMalignant pleural effusions 39 (39.8\%), TB pleural effusions 39 (39.8\%), parapneumonic pleural effusions 20 (20.4\%). In tuberculous group, the mean of ADA level was $122.4 \pm 53.3$ UI/L, malignant effusion it was $59.7 \pm 31.7 \mathrm{UI} / \mathrm{L}(\mathrm{p}=0.0001)$ parapneumonic effusions $96 \pm 48 \mathrm{UI} / \mathrm{L}$. Fluid ADA in 24/39 of malignant effusions and 18/20 parapneumonic effusions was above diagnostic cut-off $40 \mathrm{UI} / \mathrm{L}$; in four of 39 tuberculous pleurisy ADA was under 40 UI/L. Mean ADA level was greater in patients $<35$ years old $141.9 \pm 46.6 \mathrm{UI} / \mathrm{L}$. No patients of age $<35$ years had ADA level under $40 \mathrm{UI} / \mathrm{L}$. The sensitivity of $\mathrm{ADA}$ in $\mathrm{TB}$ effusions at cut-off $>40 \mathrm{U} / \mathrm{L}$ was $89.7 \%$, specificity $28.8 \%$ and PPV $54.5 \%$. In countries with low incidence of TB, the diagnostic cut-off value of ADA level have to be different from countries with high incidence. The best cut-off value of pleural fluid ADA level in diagnosis of TB was $95 \mathrm{UI} / \mathrm{L}$. In many cases, ADA levels in non-tuberculous exudative pleural effusions exceeded the cut-off values of tubercular disease (40 UI/L). Adenosine deaminase level of less than $40 \mathrm{U} / \mathrm{L}$ in patients $<35$ years excludes the tubercular aetiology of exudative pleural effusion.

D Arnold et al(29) studied patients presenting with an undiagnosed pleural effusion to a tertiary pleural centre in South-West England over a 3-year period were prospectively recruited to a pleural biomarker study, in which baseline pleural fluid samples were collected and stored. Samples from consecutive patients with robust 12-month followup data and confirmed diagnoses were sent for ADA analysis. Of 338 patients enrolled, 7 had confirmed tuberculous pleural effusion (2\%). All 7 TB effusions were lymphocyte predominant with a median ADA of 72.0 IU/L (range- 26.7 to 91.5) compared to a population median of $12.0 \mathrm{IU} / \mathrm{L}$ (range0.3 to 568.4). Using the established cut-off of $35 \mathrm{IU} / \mathrm{L}, \mathrm{ADA}$ was shown to have a Negative Predictive Value (NPV) of 99.7\% (95\% CI; 98.2 - 99.9\%) for the exclusion of TB and sensitivity of $85.7 \%$ (95\% CI; $42.2-97.6 \%$ ) with an area under the curve of $0.88(95 \% \mathrm{CI} ; 0.732-1.000)$. In the context of a lymphocytic effusion, an ADA over $35 \mathrm{IU} / \mathrm{L}$ had a sensitivity and positive predictive value of $85.7 \%$ (95\% CI; 42.2 - $97.6 \%$ ).

This is the first study examining the diagnostic utility of pleural fluid ADA in a low TB incidence area. The chance of an effusion with an ADA under 35 IU/L being of tuberculous aetiology was negligible and empirical anti-TB therapy could be avoided in such cases. A pleural ADA of over $35 \mathrm{IU} / \mathrm{L}$ in lymphocyte-predominant pleural fluid gives a strong suspicion of tuberculous aetiology. In patients who are unsuitable for more invasive procedures, this could be used as an indication to start therapy.

G. Anitha et al(30) included 80 cases of pleural effusion, out of which 50 were of tubercular pleural effusion. Increase in ADA levels was found to be significant in these cases compared to non-tubercular effusions.

Taneja Khushbu et al(31) evaluated ADA activity in pleural fluid for tubercular pleuritis. They found sensitivity of ADA in diagnosing tubercular effusions was $93.75 \%$. They suggested that all cases of lymphocytic effusions should be screened for ADA levels to exclude tubercular effusion.

Biswas B et al(32) found $77.8 \%$ of pleural effusions with ADA more than $40 \mathrm{U} / \mathrm{L}$ were diagnosed as tubercular effusion on pleural biopsy or culture or both, but $22.2 \%$ cases of biopsy proven tuberculosis had ADA levels less than $40 \mathrm{U} / \mathrm{L}$. However, all patients with high ADA levels above $81 \mathrm{U} / \mathrm{L}$ were diagnosed as tubercular effusions.

Pleural fluid ADA has long been used as a marker for tuberculous pleurisy. Levels of ADA in pleural fluid $>40 \mathrm{IU} / \mathrm{L}$ can indicate pleural tuberculosis with sensitivity (81 - 100\%) and specificity $(83-100 \%) .(33,34,35)$ The false positive cases in the literature are mainly due to empyemas, lymphomas, malignant diseases and other aetiologies, such as parapneumonic or collagen vascular disease. $(34,35,36)$ Examination of those studies reveals that pleural fluids of any cell type predominance were included. As in this study, previous reports have found increased ADA levels in patients with complicated parapneumonic effusions, wherein the immune response involves polymorphonuclear cells and macrophages rather than lymphocytes.

In present study, the negative predictive value of the ADA test was very high. The sensitivity and specificity of ADA depends on the prevalence of tuberculosis in the population. With the decline in the prevalence of tuberculous pleural effusion in some areas the positive predictive value of pleural fluid ADA also declines, but the negative predictive value remains high. Therefore, the measurement of the pleural fluid ADA level is an excellent test to rule out a tuberculous aetiology of lymphocytic pleural effusions irrespective of the rate of prevalence of the disease.

This study assesses the usefulness of ADA measurement in lymphocytic effusions. This practice resembles clinical decision making, where tuberculosis is most commonly suspected only in lymphocytic effusions. If we restrict this study to lymphocytic pleural effusion, false positives would be rare $(<2 \%)$. An elevated pleural fluid ADA level in countries with a high prevalence of tuberculous pleural effusions as in India has a high degree of specificity for tuberculous pleuritis, which makes it an integral part of the diagnostic workup of lymphocyte-rich pleural effusions. In areas where the prevalence of disease is low, there is a higher likelihood of false positive test results, and this can lead to the unnecessary administration of antituberculous therapy or a delay in making an alternative diagnosis such as malignancy. Thus far, high ADA levels in lymphocytic effusions should be looked on as a screening test to guide further diagnostic tests such as closed pleural biopsy.

\section{CONCLUSION}

Present study had a male preponderance with tubercular effusion in the young and middle aged (20 - 50 yrs.) and those 
with malignant and effusions due to cardiac failure in the elderly (50 - 80 yrs.).

The yield of sputum in the diagnosis of tuberculosis was very low and also the diagnostic yield of pleural fluid ZN stain.

Pleural biopsy also failed to give the diagnosis in all cases of tuberculosis and malignancy with a positivity rate of $60 \%$ $80 \%$ in tubercular effusions and $50 \%-85 \%$ in malignant effusions.

But all cases of tubercular effusion had ADA levels above $41.5 \mathrm{U} / \mathrm{L}$ and the mean ADA level was $73.92 \mathrm{U} / \mathrm{L}$.

We conclude that the sensitivity and specificity, positive predictive value and negative predictive value of ADA at a cut-off value of $41.5 \mathrm{U} / \mathrm{L}$ for tuberculous effusions is $100 \%$, $87 \%, 90 \%, 100 \%$ respectively.

\section{REFERENCES}

[1] Paul M. The diagnosis of tubercular pleural effusion. British Journal Dis of Chest 1979;53(86).

[2] Deshmukh MD, Virdi SS. Pleural punch biopsy in tubercular pleural effusion - to find out comparative value of single and multiple specimen's. Ind J Tub 1972;19:95.

[3] Giusti G. Adenosine deaminase. In: Bergmeyer HU. edr. Methods of enzymatic analysis. $2^{\text {nd }}$ edn. Vol. 2. New York: Academic Press inc., 1974:1092-9.

[4] Light RW, Erozan YS, Ball WC. Cells in pleural fluid. Their value in defferential diagnosis. Arch Int Med 1973;132(6):854-60.

[5] Keshmiri M, Hashemzadeh M. Use of cholesterol in differentiating of exudative and transudative pleural effusions. Medical Journal of the Islamic Republic of Iran 1997;11(3):187-90.

[6] Fine NL, Smith LR, Sheedy PF. Frequency of pleural effusions in mycoplasma and viral pneumonias. $\mathrm{N}$ Engl J Med 1970;283(15):790-3.

[7] Yam LT. Diagnostic significance of lymphocytes in pleural effusions. Ann Intern Med 1967;66(5):972-82.

[8] Light RW. Pleural Diseases. Tuberculous pleural effusion. $5^{\text {th }}$ edn. Philadelphia: Lippincott Williams and Wilkins 2007: p. 217.

[9] Grunze H. The comparative diagnostic accuracy, efficiency and specificity of cytologic techniques used in the diagnosis of malignant neoplasm in serous effusions of the pleural and pericardial cavities. Acta Cytol 1964;8:150-64.

[10] Dekker A, Bupp PA. Cytology of serous effusions. An investigation into the usefulness of cell blocks versus smears. Am J Clin Pathol 1978;70(6):855-60.

[11] Goto M, Noguchi Y, Koyama H, et al. Diagnostic value of adenosine deaminase in tuberculous pleural effusion: a meta-analysis. Ann Clin Biochem 2003; (40 Pt 4):374-81.

[12] Riantawan P, Chaowalit P, Wongsangiem M, et al. Diagnostic value of pleural fluid adenosine deaminase in tuberculous pleuritis with reference to HIV coinfection and a Bayesian analysis. Chest 1999;116(1):97-103.

[13] Chung JH, Kim YS, Kim SI, et al. The diagnostic value of the adenosine deaminase activity in the pleural fluid of renal transplant patients with tuberculous pleural effusion. Yonsei Med J 2004;45(4):661-4.
[14] Piras MA, Gakis C, Budroni M, et al. Adenosine deaminase activity in pleural effusions an aid to differential diagnosis. Br Med J 1978;2(6154):1751-2.

[15] Sinha PK, Sinha BB, Sinha AR. Diagnosing tuberculous pleural effusion: comparative sensitivity of mycobacterial culture, histopathology and adenosine deaminase activity. JAPI 1985;33(10):644-5.

[16] Prasad R. Adenosine deaminase level in pleural fluide. Annual reports of Indian J of TB and Chest Diseases 1989.

[17] Ferrer JS, Munoz XG, Orriols RM, et al. Evolution of idiopathic pleural effusion: a prospective, long-term follow-up study. Chest 1996;109(6):1508-13.

[18] Maldhure BR. Pleural biopsy and Adenosine Deaminase in pleural fluide for the diagnosis of tubercular pleural effusion. Ind J Tub 1993:161-5.

[19] Valdes L, Jose SE, Alvarez D, et al. Adenosine deaminase (ADA) isoenzyme analysis in pleural effusion: diagnostic role and relevance to the origin of increased ADA in tuberculous pleurisy. Eur Respir J 1996;9(4):747-51.

[20] Al-Shammary FJ. Adenosine Deaminase activity in serum and pleural effusions of tuberculosis and nontuberculosis patients. Biochemistry and Molecular Biology International 1997;43(4):763-79.

[21] Perez-Rodriguez E, Castro JD. The use of Adenosine Deaminase and adenosine deainase isoenzymes in the diagnosis of tuberculous pleuritis. Curr Opin Pulm Med 2000;6(4):259-66.

[22] Sharma SK, Suresh V, Mohan A, et al. A prospective study of sensitivity and specificity of adenosine deaminase estimation in diagnosis of tuberculosis pleural effusion. Indian J Chest Dis Allied Sci 2001;43(3):149-55.

[23] Castro JD, Nuevo DG, Pérez-Rodríguez E, et al. Diagnostic value of adenosine deaminase in nontuberculous lymphocytic pleural effusion. Eur Resp J 2003;21(2):220-4.

[24] Zahir TS, Salehinia H. Evaluation of diagnostic value of measuring adenosine deaminase in pleural effusion to differentiate between tuberculosis and malignancy by comparision with pleural biopsy. RJMS 2007;14(54):63-9.

[25] Baba K, Hoosen AA, Langeland N, et al. Adenosine deaminase activity is a sensitive marker for the diagnosis of tuberculous pleuritis in patients with very low CD4 counts. PLoS ONE 2008;3(7): e2788.

[26] Jadhav AA, Bardapurkar JS. Diagnostic value of adenosine deaminase to differentiate exudates and transudates. Indian Journal of Physiol Pharmacol 2007;51(2):170-4.

[27] Dhital KR, Acharya R, Bhandari R, et al. Clinical profile of patients with pleural effusion admitted to KMCTH. KUMJ 2009;7(28):438-44.

[28] Kapisyzi P, Argjiri D, Aliko A, et al. The use of different cut off values of ADA liquid level in the diagnosis of tuberculous pleurisy in countries with different incidence of tuberculosis. Chest 2011;140(4).

[29] Arnold D, Bhatnagar R, Fairbanks L, et al. S115 Pleural fluid ADA in the diagnosis of tubercular pleural effusions in a low incidence population. Thorax 2014;69(Suppl 2). 
[30] Anitha G, Mahaboob RS, Obulesu G. Study of ADA levels in the diagnosis of Tuberculous pleural effusion. IAIM 2016;3(7):353-7.

[31] Khushbu T, Lal GM. Evaluation of ADA activity in pleural fluid for Tubercular pleuritis. International Journal of Contemporary Medical Research 2016;3(10):3083-5.

[32] Biswas B, Sharma SK, Negi RS, et al. Pleural effusion: role of pleural fluid cytology, ADA level and pleural biopsy in diagnosis. J Cytology 2016;33(3):159-62.

[33] Ribera E, Martı́nez-Va'zquez JM, Ocana L, et al. Gamma interferon and adenosine deaminase in pleuritis. Med Clin (Barc) 1990;94(10):364-7.
[34] Valdes L, Jose' ES, Alvarez D, et al. Diagnosis of tuberculous pleuresy using the biologic parameters adenosine deaminase, lysozime and interferon gamma. Chest 1993;103(2):458-65.

[35] Muranhisi H, Nakashima M, Hirano $H$, et al. Simultaneous measurements of adenosine deaminase activity and tuberculostearic acid in pleural effusion for the diagnosis of tuberculous pleuritis. Intern Med 1992;31(6):752-5.

[36] Ocana L, Ribera E, Martı́nez-Va'zquez JM, et al. Adenosine deaminase activity in rheumatoid pleural effusion. Ann Rheum Dis 1988;47(5):394-7. 